\title{
Repeated-task Canadian traveler problem
}

\author{
Zahy Bnaya and Ariel Felner \\ Information Systems Engineering \\ Deutsche Telekom Labs \\ Ben-Gurion University \\ Be'er-Sheva, Israel \\ \{zahy,felner\}@bgu.ac.il
}

\author{
Solomon Eyal Shimony, Dror Fried, Olga Maksin \\ Computer Science, \\ Ben-Gurion University \\ Be'er-Sheva, Isrel \\ $\{$ shimony,dfried,omaksin\}@cs.bgu.ac.il
}

\begin{abstract}
In the Canadian Traveler Problem (CTP) a traveling agent is given a graph, where some of the edges may be blocked, with a known probability. A solution for CTP is a policy, that has the smallest expected traversal cost. CTP is intractable. Previous work has focused on the case of a single agent. We generalize CTP to a repeated task version where a number of agents need to travel to the same goal, minimizing their combined travel cost. We provide optimal algorithms for the special case of disjoint path graphs. Based on a previous UCT-based approach for the single agent case, a framework is developed for the multi-agent case and four variants are given - two of which are based on the results for disjoint-path graphs. Empirical results show the benefits of the suggested framework and the resulting heuristics. For small graphs where we could compare to optimal policies, our approach achieves near-optimal results at only a fraction of the computation cost.
\end{abstract}

\section{Introduction}

The Canadian Traveler Problem (CTP) (Nikolova and Karger 2008; Papadimitriou and Yannakakis 1991; Bar-Noy and Schieber 1991) is a navigation problem where traveling agent receives a graph as input and needs to travel from its initial location to a given goal location. The complication in CTP is that some edges of the graph may be blocked with a known probability. The basic action in the CTP is a move action along an edge of the graph. Moves incur costs. After arriving at a node, the agent can sense its incident edges at no cost. The task is to minimize the travel cost of reaching the goal. Since some of the graph's edges may be blocked, a simple search for a path does not work; a solution is a contingent plan, or policy, that has the smallest expected traversal cost.

In the basic version of the CTP, a single agent is assumed. However, in many realistic settings the problem needs to be solved for a group of agents, requiring minimization of the combined travel cost of all agents. For example, think of an owner of a fleet of trucks, who has to move the trucks from a single source to a single destination. In this paper, we generalize CTP to its multi-agent repeated-task setting, that assumes that the agents traverse the graph sequentially. We also briefly examine the interleaved version, where agents are allowed to traverse the graph concurrently. Single-agent CTP is intractable (Papadimitriou and Yannakakis 1991).
Having multiple agents further increases the size of the statespace, which makes finding an optimal solution even harder and is also intractable. Therefore, we cannot find optimal solutions efficiently in general. However, we provide efficient optimal solutions for special case graphs which are then used to generate heuristics for the general case. These heuristics are tested empirically against baselines developed for single and multi-agent. Experimental results show that our heuristics appear to improve the expected travel cost, and are not too far from the optimal for small graphs.

\section{Variants of the Canadian Traveler Problem}

In the single agent version of CTP, a traveling agent is given a connected weighted graph $G=(V, E)$, a source vertex $(s \in V)$, and a target vertex $(t \in V)$. The input graph $G$ may undergo changes, that are not known to the agent, before the agent begins to act, but remains fixed subsequently. In particular, some of the edges in $E$ may become blocked and thus untraversable. Each edge $e \in E$ has a weight, $w(e)$, and is blocked with a known blocking probability $p(e)$, or traversable with probability $q(e)=1-p(e)$. The agent can perform move actions along an unblocked edge which incurs a travel cost equal to $w(e)$. The status of an edge (blocked or traversable) is revealed to the agent, only after the agent reaches a vertex incident to that edge.

The task of the agent is to travel from $s$ to $t$ with minimal total travel cost. As the exact travel cost is uncertain until the end, a solution to CTP is a traveling strategy (policy) which yields a small (ideally optimal) expected travel cost.

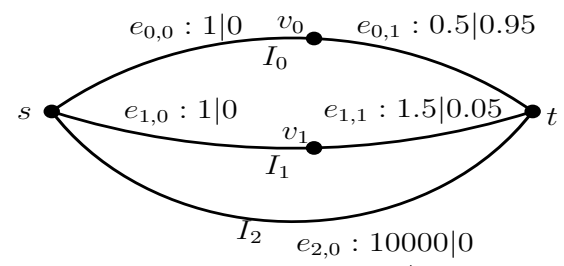

Figure 1: CTP example. Edge label $w \mid p$ denotes edges cost $w$, blocking probability $p$.

To illustrate CTP, consider figure 1 with unknown edges $e_{0,1}$ and $e_{1,1}$. If $e_{0,1}$ is traversable, the cheapest path is $\left(s, v_{0}, t\right)$ with cost 1.5 , but if $e_{0,1}$ is blocked, and $e_{1,1}$ is traversable, the cheapest path is $\left(s, v_{1}, t\right)$ with cost 2.5. Taking into account the blocking events $\left(e_{0,1}\right.$ is likely to be blocked, and its status can only be observed from $v_{0}$ ), the 
optimal policy is to try $v_{1}$ first, then if $e_{1,1}$ is blocked go to $v_{0}$; finally if $e_{0,1}$ is also blocked, reach $t$ though path $I_{2}$.

\section{Multi-agent CTP}

In this paper, we generalize CTP to its multi-agent case. where several $(n)$ agents operate in the given graph. We assume that the agents are fully cooperative and aim to minimize their total travel cost. In addition, we assume a communication paradigm of full knowledge sharing. That is, any new information discovered by an agent (e.g., whether an edge is blocked or traversable) is immediately made known to all other agents. This assumption is equivalent to having a centralized control of all agents. In our trucks example, the trucks have full communication with the owner, who, on his part, is interested in minimizing the expected total cost of the entire fleet. ${ }^{1}$

Repeated task CTP In the Repeated task multi-agent CTP (called CTP-REP $(n)$ for short) there is only one active agent at a time. All other agents are inactive until the currently active agent reaches $t$. An agent that reaches $t$ becomes inactive again (is "out of the game"), and can make no additional actions or observations. The goal is a natural extension of single-agent CTP: all $n$ agents begin at $s$, and must reach $t$. We need to find a policy for the agents that minimizes the expected total travel cost of reaching this goal. Equivalently, this can be seen as repeating the single-agent CTP task $n$ times, but in each case based on observations gathered in all previous traversals. Similar combinations of multi-agent and repeated task navigation were presented by (Meshulam, Felner, and Kraus 2005) but for for the case of completely unknown graphs (as opposed to the CTP framework here).

Interleaved repeated-task CTP In the more general interleaved action multi-agent CTP (called CTP-MULTI $(n)$ for short), the agents make their moves in a round-robin fashion. No-op actions, where an agent does not move in a specific turn, with a cost of zero, are permitted. Note that one could consider an alternate definition of the problem, where agents move in parallel. However, in the latter setting, we can prove the following property: there exists an optimal policy where all agents but one perform a "no-op" at every step, since no cost is incurred by doing nothing (proof by policy simulation). Thus, there is no need to examine the parallel action case.

\section{Complexity of Single and Multi-Agent CTP}

Define a weather as a given assignment of \{traversable,blocked\} status to an edge. There are $2^{|E|}$ different possible weathers. Therefore, the size of the state space of CTP is $V \times 2^{|E|}$ because a state also includes the specific location of the agent. Partially Observable Markov Decision Processes (POMDP) offer a model for cost optimization under uncertainty, and CTP can be modeled as an indefinite horizon POMDP (Hansen 2007). POMDPs use the notion of a belief state to indicate knowledge about the state. In CTP, the belief state is the location of the agent coupled with the belief status about

\footnotetext{
${ }^{1}$ The treatment of other types of agents (e.g., selfish or adversarial agents) and other communications paradigms (e.g., message exchanging) is beyond the scope of this paper.
}

the edges (the hidden variables). One could specify edge variables as ternary variables with domain: \{traversable, blocked, unknown $\}$, thus the belief space size is $O\left(|V| 3^{|E|}\right)$ (where is the agent, what do we know about the status of each edge). An optimal policy for this POMDP, is a mapping from the belief space into the set of actions, that minimizes the expected travel cost.

Solving POMDPs is PSPACE-hard (in the number of states) in the general case. However, because the only source of uncertainty in CTP is in the initial belief state and not in the outcome of the actions, CTP is actually a special case of the simpler deterministic POMDP (Littman 1996; Bonet 2009), which can be solved in time polynomial in the size of the state space. Unfortunately these results do not help solving CTP, because the size of the CTP state space $\left(O\left(|V| 2^{|E|}\right)\right)$ is exponential in the size of the input graph. In fact CTP is known to be \#P-hard (Papadimitriou and Yannakakis 1991), and some variants are known to be PSPACEcomplete. Multi-agent CTP is potentially harder. The size of the state space is now $O\left(|V|^{n} 2^{|E|}\right)$ for $\operatorname{CTP}-\operatorname{MULTI}(n)$ as we need to specify the locations of $n$ agents for each state, and $O\left(n|V| 2^{|E|}\right)$ for $\operatorname{CTP}-\operatorname{REP}(n)$ because we have $n$ phases, one per agent.

\section{Theoretical Results on Disjoint Paths}

We restrict the theoretical analysis in this paper to disjointpath graphs (CTP-DISJ) such as that shown in figure 1 because every departure from this topology makes the analysis prohibitively complex, even for single-agent CTP. For example, adding even one edge to an instance of CTP-DISJ, either between nodes in different paths or from such a node directly to $t$, violates the conditions under which committing policies (see below) are optimal. The situation is made even more complex in multi-agent CTP. Nevertheless, our theoretical results on this restricted version provide insight for the general case, as shown below.

A CTP-DISJ graph has $k \geq 2$ paths, denoted by $I_{0}, \cdots, I_{k-1}$. We assume w.l.o.g. that at least one path is known to be traversable. Otherwise, we can add a traversable path consisting of one edge with a finite, but very large cost, between $s$ and $t$. If the agent reveals that all other paths are blocked, it can backtrack and follow this additional edge, which can be thought of as a rescue choice, such as call an helicopter, that the agent must take if no regular path to the goal exists.

The length $r_{i}$ of each path $I_{i}$, is the number of the edges of $I_{i}$. The edges of path $I_{i}$ starting from $s$ are denoted by $e_{i, j}$ for $0 \leq j<r_{i}$, (see Fig. 1). For a path $I_{i}$, and an edge $e_{i, j}$, let $W_{i, j}=\sum_{l<j} w\left(e_{i, l}\right)$ be the cost of the path $I_{i}$ up to edge $e_{i, j}$ without including $e_{i, j}$, and let $W_{i}=W_{i, r_{i}}$ be the cost of the entire path $I_{i}$. We also define $Q_{i}$ to be the probability of path $I_{i}$ being unblocked; thus $Q_{i}=\prod_{l<r_{i}} q\left(e_{i, l}\right)$.

\section{Single-Agent CTP on disjoint paths}

The analysis for single-agent CTP-DISJ is from (Bnaya, Felner, and Shimony 2009; Nikolova and Karger 2008) and repeated here as the basis for the multi-agent case. Reasonable policies are policies that do not involve the agent walking around without making headway and without obtaining new information. An optimal policy must also be reason- 
able. Two "macro actions" are defined in terms of which all reasonable policies on disjoint-paths graph can be specified. Both macro actions are defined for an agent situated at $s$.

Definition 1 For path $I_{i}$, macro action $T R Y(i)$ is to move forward along path $I_{i}$ until reaching $t$; if a blocked edge is encountered, the agent returns along the path and stops at $s$. An agent performing a TRY action on a path is said to be trying the path.

Definition 2 For path $I_{i}$, and an edge $e_{i, j}$, macro action $I N V(i, j)$ (meaning investigate) is to move forward along path $I_{i}$ until reaching (without crossing) $e_{i, j}$, or a blocked edge, whichever occurs first. In either case the agent returns to $s$.

For path $I_{i}$, denote by $B C\left(I_{i}\right)$ the random variable representing the backtracking cost of path $I_{i}$ : the cost of traveling path $I_{i}$, finding $I_{i}$ blocked, and returning to $s$. Since the path can be blocked anywhere, and the cost to reach edge $e_{i, j}$ is $W_{i, j}$, the expected backtracking cost is:

$$
E\left[B C\left(I_{i}\right)\right]=2 \sum_{j<r_{i}} W_{i, j} p\left(e_{i, j}\right) \prod_{l<j} q\left(e_{i, l}\right)
$$

Denote the expected cost of $T R Y\left(I_{i}\right)$ by $E\left[T R Y\left(I_{i}\right)\right]$ :

$$
E\left[T R Y\left(I_{i}\right)\right]=Q_{i} W_{i}+E\left[B C\left(I_{i}\right)\right]
$$

A policy which consists only of $T R Y$ actions, but never uses $I N V$ actions, (that is, only backtracks if a blocked edge is revealed), is called committing. Since a $T R Y$ macro action either reaches $t$ or finds a path blocked, it never makes sense to try the same path more than once, and thus all such committing policies can be represented by an order of paths to be tried. Let $M$ be an instance of CTP-DISJ, and $\chi_{M}^{*}$ be a committing policy for $M$ in which the agent tries the paths in a non decreasing order of $\frac{E\left[T R Y\left(I_{i}\right)\right]}{Q_{i}}$. Assuming without loss of generality that $\frac{E\left[T R Y\left(I_{i}\right)\right]}{Q_{i}}$ are all different, and thus $\chi_{M}^{*}$ is unique. Then the following theorem (Bnaya, Felner, and Shimony 2009) holds:

Theorem $1 \chi_{M}^{*}$ is an optimal policy for $M$.

\section{Repeated CTP in disjoint-path graphs}

We now adapt these results to Repeated CTP with $n$ agents on disjoint paths graph (CTP-DISJ-REP $(n))$. Let $M$ be an instance of CTP-DISJ-REP $(n)$ with $k$ paths. Note that any reasonable policy in $M$ can be represented using only $T R Y$ and $I N V$ macro actions as follows. Let $T R Y(l, i)$ be the action in which agent $A_{l}$ tries path $I_{i}$, and let $I N V(l, i, j)$ be the action in which agent $A_{l}$ performs $I N V(i, j)$. A policy for an agent $A_{i}$ is committing if $A_{i}$ executes only $T R Y$ actions. Likewise, a policy (for a set of agents) is committing if it consists only of $T R Y$ actions for all agents. It is non-trivial to show that in repeated CTP, TRY actions suffice for optimality - this requires definition of the restricted followers-commiting policies, discussed next.

Let $\pi$ be a policy for $M$, where whenever $A_{0}$ reaches $t$ through path $I_{i}$, for some $i<k$, the agents $A_{1}, \cdots A_{n-1}$ traverse $I_{i}$ as well. A policy $\pi$ with this property is called a followers-committing policy, and the agents $A_{1}, \cdots A_{n-1}$ are said to follow $A_{0}$ in $\pi$.

Denote by $T R Y_{n}\left(I_{i}\right)$ the action $T R Y(0, i)$ executed in a followers-committing policy in CTP-DISJ-REP $(n)$. The results of such actions are either that $A_{0}$ traverses $I_{i}$ to $t$, and the other agents follow $A_{0}$ on $I_{i}$, or that $A_{0}$ finds $I_{i}$ blocked and backtracks to $s$ (other agents staying idle). Let $E\left[T R Y_{n}\left(I_{i}\right)\right]$ be the expected cost of $T R Y_{n}\left(I_{i}\right)$. We get:

$$
E\left[T R Y_{n}\left(I_{i}\right)\right]=n Q_{i} W_{i}+E\left[B C\left(I_{i}\right)\right]
$$

Let $<_{*}$ be the following order on the $k$ paths: $l<_{*} j$ if and only if $\frac{E\left[T R Y_{n}\left(I_{i}\right)\right]}{Q_{i}}<\frac{E\left[T R Y_{n}\left(I_{j}\right)\right]}{Q_{j}}$, assuming again w.l.o.g. that all of these fractions are different. Let $\sigma_{*}$ be the permutation on $\{0, \cdots, k-1\}$ which is induced by $<_{*}$.

Definition 3 Let $\pi_{M}^{*}$ be the followers-committing policy where $A_{0}$ executes the committing policy of trying the paths by increasing order of $<_{*}$, and $A_{1}, \cdots, A_{n-1}$ follow $A_{0}$.

We show that $\pi_{M}^{*}$ is optimal, but first need to show that $\pi_{M}^{*}$ is optimal among all followers-committing policies. Denote the expected cost of policy $\pi$ by $c(\pi)$.

Lemma 1 Let $\pi$ be a followers-committing policy for $M$. Then $c\left(\pi_{M}^{*}\right) \leq c(\pi)$.

Proof outline: Note that any followers-committing policy $\pi$ for an instance $M$ of CTP-DISJ-REP $(n)$ can be re-cast as an equivalent CTP-DISJ problem instance $M^{\prime}$, where an extra cost of $(n-1) W_{i}$ is incurred once that agent reaches $t$ through path $I_{i}$, as follows. $M^{\prime}$ is an extension of $M$, where we add at the end of each path $I_{i}$, an additional traversable edge $e_{i, r_{i}}$ incident on $t$, with a cost of $(n-1) W_{i}$. Since, in a followers-committing policy for $M$, all agents follow the first agent, thus each agent incurs a cost of $W_{i}$, there is a trivial bijection $F$ between followers-committing policies in $M$, and committing policies in $M^{\prime}$, that preserves expected costs, such that $F\left(\pi_{M}^{*}\right)=\chi_{M^{\prime}}^{*}$. Now suppose that $\pi$ is a followers-committing policy for $M$. Then by theorem 1 , $c\left(F\left(\pi_{M}^{*}\right)\right) \leq c(F(\pi))$, hence $c\left(\pi_{M}^{*}\right) \leq c(\pi)$.

Theorem $2 \pi_{M}^{*}$ is an optimal policy for $M$.

Proof outline: By induction on $n$. For $n=1$, we have an instance of CTP-DISJ-REP(1), $M^{\prime}$, which is also an instance of CTP-DISJ. Hence, by theorem $1, \pi_{M^{\prime}}^{*}$ is optimal.

We now assume inductively that $\pi_{M}^{*}$ is an optimal policy for any instance of CTP-DISJ-REP $(n-1)$, and show that this property holds for all instances of CTP-DISJ-REP $(n)$ as well. Let $M$ be an instance of CTP-DISJ-REP $(n)$. Given a committing policy $\bar{\pi}$ for $M$, denote by $\sigma_{\bar{\pi}}$ the permutation on $\{0, \cdots, k-1\}$ which is induced by the order in which $A_{0}$ tries the paths in $\bar{\pi}$. For $\sigma$, and $\nu$, permutations on $\{0, \cdots, k-1\}$, let $d(\sigma, \nu)$ be the "Euclidean" distance between $\sigma$, and $\nu$ (the Euclidean distance is known as $\left.d(\sigma, \nu) \stackrel{\text { def }}{=} \sqrt{\sum_{i<k}(\sigma(i)-\nu(i))^{2}}\right)$.

A CTP instance $M$ can be represented as a directed action/outcome AND/OR tree $T_{M}$, and a policy $\pi$ can be represented by an AND-subtree $T_{\pi}$ of $T_{M}$. For a general policy $\pi^{\prime}$, let an $I N V$-edge be any action-edge in $T_{\pi^{\prime}}$ where the action taken by the agent is $I N V$, and let $I N V\left(T_{\pi^{\prime}}\right)$ be the number of $I N V$-edges in $T_{\pi^{\prime}}$. Since $\pi_{M}^{*}$ is committing, then $I N V\left(T_{\pi_{M}^{*}}\right)=0$.

Let $\pi$ be an optimal policy for $M$, with $I N V\left(T_{\pi}\right)$ as small as possible, and such that if $\operatorname{INV}\left(T_{\pi}\right)=0$, then $d\left(\sigma_{\pi}, \sigma_{*}\right)$ is minimal. There are two cases:

(1:) $\pi$ is committing. Then $\operatorname{INV}\left(T_{\pi}\right)=0$. We may assume that $A_{0}$ tries the paths in $\pi$ in the order of $\left\{I_{0}, I_{1}, \cdots I_{k-1}\right\}$. By the induction assumption we have 
that $A_{2}, \cdots A_{n-1}$ follow $A_{1}$ in $\pi$. If $A_{1}$ also follows $A_{0}$, then $\pi$ is a followers-committing policy, and by lemma 1 , $c\left(\pi_{M}^{*}\right) \leq c(\pi)$, hence $\pi_{M}^{*}$ is an optimal policy for $M$. If $A_{1}$ does not follow $A_{0}$, we can show that there is a path $u<k-1$ such that $u+1<_{*} u$. We then define a policy $\pi^{\prime}$ which is the same as $\pi$, except that $I_{u+1}$ is tried right before $I_{u}$. As $\pi^{\prime}$ is committing as well, $\operatorname{INV}\left(T_{\pi^{\prime}}\right)=\operatorname{INV}\left(T_{\pi}\right)=$ 0 . Then we can show that $\pi^{\prime}$ is an optimal policy for $M$, and $d\left(\sigma_{\pi^{\prime}}, \sigma_{*}\right)<d\left(\sigma_{\pi}, \sigma_{*}\right)$, contradicting the minimality of $d\left(\sigma_{\pi}, \sigma_{*}\right)$ among the committing optimal policies of $M$.

(2:) $\pi$ is not committing. Then $I N V\left(T_{\pi}\right)>0$. We can then show that $T_{\pi}$ contains a subtree, $T$, with only one $I N V$-edge, and define a policy $\pi^{\prime}$ which is obtained from $\pi$ by replacing $T$ with another tree, $T^{\prime}$, which has no $I N V$-edges at all. We then show that $\pi^{\prime}$ is optimal and $I N V\left(T_{\pi^{\prime}}\right)<I N V\left(T_{\pi}\right)$, contradicting the minimality of $I N V\left(T_{\pi}\right)$ among the optimal policies of $M . \square$

Example 1 Consider Fig. 1. We have $\frac{E\left[T R Y_{1}\left(I_{0}\right)\right]}{Q_{0}}=39.5$, and $\frac{E\left[T R Y_{1}\left(I_{1}\right)\right]}{Q_{1}}=2.6$. Hence by theorem 2, the optimal single agent policy is committing to try path $I_{1}$ before $I_{0}$. However, $\frac{E\left[T R Y_{38}\left(I_{0}\right)\right]}{Q_{0}}=95$, and $\frac{E\left[T R Y_{38}\left(I_{1}\right)\right]}{Q_{1}}=95.1$, hence for $n \geq 38$ agents, the optimal policy is for the first agent, to try path $I_{0}$ before $I_{1}$, and for the other agents to follow the first agent's path to $t$.

Interleaved-action CTP in disjoint-path graphs We briefly consider interleaved action CTP in disjoint-path graphs (CTP-DISJ-MULTI $(n)$ ). Since agents can start moving before the first active agent has reached $t$, it is by no means clear that the optimal policy can be described using only $T R Y$ and $I N V$ macro actions. In fact, it is easy to see that for more general graphs, the optimal policy requires interleaved actions. For example, adding a (certainly traversible) path that costs 100 from $v_{1}$ to $t$ in Fig. 1, the optimal 2-agent policy is to send the first agent to $v_{1}$, and if $e_{1,1}$ is blocked, send the second agent to $v_{0}$ to check $e_{0,1}$, while the first agent waits at $v_{1}$.

Since for disjoint paths this type of scenario cannot occur, we are led to suspect that there is no advantage to having more than one active agent at a time in this topology. We have checked this empirically by generating the optimal policies (using value iteration) with and without interleaved actions for small randomly generated problem instances. From hundreds of such non disjoint-path instances, more than $10 \%$ of the cases required interleaved actions to achieve the optimal policy. Conversely, in all of over a thousand such disjoint-path graph instances, the optimal policy for CTP$\operatorname{DISJ-REP}(n)$ was also optimal for CTP-DISJ-MULTI $(n)$. Hence we state the following:

Conjecture: Any optimal policy for CTP-DISJ-REP $(n)$ is also optimal for CTP-DISJ-MULTI $(n)$.

\section{Heuristics for repeated-task CTP}

For general graphs, the size of the tree of the optimal policy, and the time to compute it, are exponential in the number of unknown edges even for single-agent CTP. Thus, such policies can be computed and described only for very small graphs. We thus examine non-optimal policies suggested for CTP in the literature. The optimistic policy (OPT) (Bnaya, Felner, and Shimony 2009; Eyerich, Keller, and Helmert
2010), is based on the "free-space assumption" (commonly used in robotics) which assumes that all unknown edges are traversable. The agent computes the shortest path under this optimistic assumption, and begins to traverse it. The shortest path from the current location to the goal is re-computed every time an edge on the currently traveresed path is found to be blocked. OPT is fast, but far from optimal in expected cost.

(Eyerich, Keller, and Helmert 2010) performed systematic comparison between sampling-based schemes. Their best sampling method (denoted as CTP-UCT) was based on the UCT scheme (Kocsis and Szepesvári 2006). They showed that CTP-UCT converges in the limit to the optimal behavior and outperforms other techniques for single agentCTP. We revisit single-agent CTP-UCT and then generalize it to the multi-agent case.

\section{UCT for Single-Agent CTP}

CTP-UCT works by performing "rollouts" as follows. For each rollout a weather (defined above) is randomized. Status of unknown edges is revealed only when the search reaches the appropriate node. Starting with the initial belief state $b_{0}$, choose an action $a_{1}$ to explore. The choice is made according to the CTP-UCT formula defined below. This results in a new belief state $b_{1}$, depending on $b_{0}$, the current weather, and the action $a_{1}$. Then, a new action is chosen, resulting in a new belief state $b_{2}$. This is repeated until we reach a belief state $b_{m}$ where the agent is located at $t$. This is a single rollout, involving a sequence of belief states $<b_{0}, b_{1} \ldots . b_{m}>$.

Let $\sigma=<b_{0}, b_{1} \ldots b_{i}>$ be a partial sequence of belief states generated in the $(\mathrm{k}+1)$-th rollout where the agent location in $b_{i}$ is not $t$. Define: (1:) $R^{k}(\sigma)$ to be the number of rollouts among the first $k$ rollouts that start with $\sigma$ (2:) $C^{k}(\sigma)$ to be the average cost incurred on these rollouts from the end of $\sigma$ to the goal.

There are several possible successor belief states $b_{1}^{\prime}, b_{2}^{\prime} \ldots$, one for each possible action $a_{1}, a_{2} \ldots$. Each successor $b_{i}^{\prime}$, when appended to $\sigma$, results in an extended sequence of belief states denoted by $\sigma_{i}$. Based on (Kocsis and Szepesvári 2006), CTP-UCT picks the action whose successor state maximizes:

$U C T\left(\sigma_{i}\right)=B \sqrt{\frac{\log R^{k}(\sigma)}{R^{k}\left(\sigma_{i}\right)}}-\operatorname{cost}\left(a_{i}\right)-C^{k}\left(\sigma_{i}\right)$

where $B>0$ is a parameter, and $\operatorname{cost}\left(a_{i}\right)$ is the cost of the edge traversed by action $a_{i}$. The idea here is to allow "exploration" initially (i.e. to sample previously little-visited actions), due to first term in the equation. Eventually, when many rollouts are performed the equation gives more weight to minimizing the expected travel cost as expressed by the last 2 terms in the equation. In order to direct the sampling better towards the goal, the value $C^{k}\left(\sigma_{i}\right)$ is initially based on the optimistic cost, based on the free-space assumption.

After completing a pre-determined number of rollouts, an agent performs the action with the minimal average cost to reach the goal, where averaging is computed based on the rollouts. This results in a new belief state actually reached by the agent, and the process (of CTP-UCT) is repeated until the agent reached the goal.

\section{Generalization of UCT to Repeated-Task CTP}

In CTP-REP, knowledge revealed to an agent is immediately shared with all other agents. All agents share a known graph 
which includes all edges known to be traversable. There are 4 different ways to generalize UCT to work for repeated CTP depending on the following attributes.

1: behavior of consecutive agents. After the first agent activates CTP-UCT there are two possible behaviors of the remaining agents. They can be reasoning agents, that is, they also active CTP-UCT. Or, they can be followers: Since the first agent has arrived at the goal, the known graph includes at least one path to the goal. The shortest such known path is followed by the remaining $n-1$ agents.

2: considering consecutive agents. An agent can be inconsiderate, ignore the remaining agents, and use the CTPUCT formula of the single agent case. By contrast, it can be considerate. In this case, the agent considers the fact that the remaining $n-1$ agents are expected to travel to the goal too and therefore their expected travel cost should be taken into account. One way to do it is by assuming that consecutive agents will follow the shortest known path. This idea is based on the theoretical results for disjoint graphs, where this policy is actually optimal. While no longer necessarily optimal for general graphs, it is used here as a heuristic. In order to implement this scheme, we modify the CTP-UCT formula by adding an additional $F^{k}$ term (stands for "future path"), which is the cost of the shortest path we expect to discover at the end of the rollout. This is multiplied by $n-1$, since $n-1$ agents are expected to follow that path as well. We therefore use the following modified UCT rule:

$$
\operatorname{UCTR}\left(\sigma_{i}\right)=U C T\left(\sigma_{i}\right)-(n-1) F^{k}\left(\sigma_{i}\right)
$$

where $F^{k}\left(\sigma_{i}\right)$ is the average over $k$ rollouts of the shortest path from $s$ to $t$ among edges known to be unblocked in belief states at the end of the rollout starting from $\sigma_{i}$. As this quantity is not well defined initially, it is initialized based on the value of the shortest path from $s$ to $t$ under the free-space assumption.

Example 1 demonstrates the difference between a considerate and an inconsiderate agent, as an inconsiderate first agent always chooses to try $I_{1}$ before $I_{0}$ no matter how many agents follow it. There are four possible combinations, which lead to four possible variants that generalize CTP-UCT as follows.

(1:) Inconsiderate, followers. In this variant, only the first agent activates CTP-UCT for choosing its actions. The first agent inconsiderately ignores the existence of the other agents and activates CTP-UCT for choosing its actions. The other agents are followers. This variant is labeled UCTR1.

(2:) Considerate, followers. The first agent activates UCTR. The other agents are followers. This variant is labeled UCTR2.

(3:) Inconsiderate, reasoning. Here, all agents are reasoning agents but they are all inconsiderate, i.e., they all activate CTP-UCT. This variant is labeled UCTR3.

(4:) Considerate, reasoning. All agents are reasoning agents and they all activate UCTR. This variant is labeled UCTR4.

\section{Empirical Evaluation}

Our experiments included instances with small number of unknown edges. This enables the evaluation of a given policy under all possible weathers and measuring the policy performance accurately as all cases have been evaluated. We experimented on problem instances based on the graph depicted in figure 2(a). In the figure, the source and target vertices are labeled $s$ and $t$, respectively. Each edge is labeled with the edge weight.

We generated two sets of instances where we randomly selected 4 and 8 unknown edges (not adjacent to $s$ ) respectively. We also uniformly select blocking-probabilities for each of the unknown edges in the range of $(0.01,0.9)$. Similar trends were observed and we only report results on the graph with 8 unknown edges.

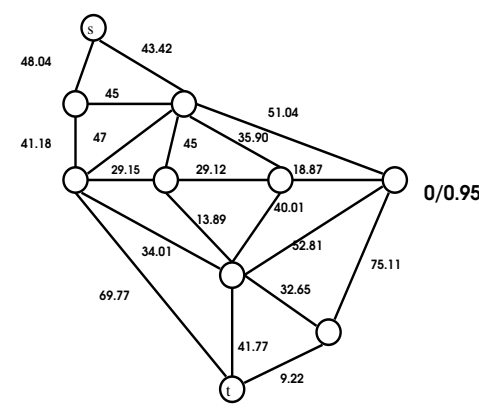

(a)

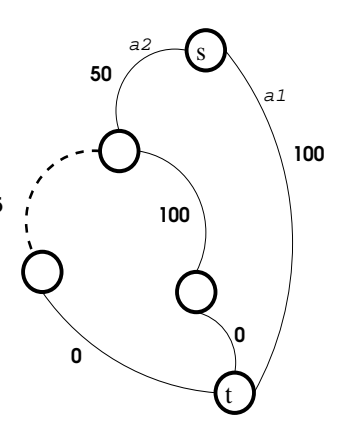

(b)
Figure 2: Our example graphs

We implemented six policies for CTP-REP. First, we computed the optimal policy using value iteration. This serves as a lower bound on the travel cost. Second, we implemented a bound we call cautious-blind (C-Blind in the tables). The cautious-blind uses only the known edges of the graph to find a shortest path from source to target and then follows it. If any unknown edges are revealed to the agent while traversing, the agent ignores this information. Cautious-blind serves only to indicate a type of "worst-case" and thus we define a range of costs that makes sense for each problem instance. Finally, the expected costs for our four UCTR variants described above are achieved by averaging simulations of the policy under each possible weather of an instance. The travel cost incurred in each simulation is weighted according to the probability of the weather. Therefore, this gives the expected cost of the policy on a given instance. UCTR variants were tried with 10000-200000 rollouts.

\begin{tabular}{|l|r|r|r|}
\hline agents & $\mathrm{n}=2$ & $\mathrm{n}=3$ & $\mathrm{n}=10$ \\
\hline Optimal & 15.45 & 35.37 & 361.00 \\
UCTR1 & 1.72 & 1.73 & 1.73 \\
UCTR2 & 1.77 & 1.84 & 1.94 \\
UCTR3 & 3.15 & 4.23 & 6.23 \\
UCTR4 & 3.25 & 4.54 & 6.34 \\
\hline
\end{tabular}

Table 1: Execution times in minutes

Table 1 presents the execution time in minutes required to generate the polices on a typical instance with 8 unknown edges. Clearly, as the number of agents, $n$, grows, computing the optimal policy consumes significantly more time than any other policy. UCTR1, and UCTR2 are both policies that are based on our "followers" principle. Thus, the execution of UCT is only performed once, for the first agent. As a result, the time it takes to calculate these policies is significantly smaller than UCTR3 and UCTR4.

We have performed a number of experiments and did not see evidence that the reasoning policies UCTR3 and UCTR4 


\begin{tabular}{|l|r|r|r|r|r|r|}
\hline & \multicolumn{3}{|c|}{ Instance 1 } & \multicolumn{3}{|c|}{ Instance 2 } \\
\hline & $\mathrm{N}=1$ & $\mathrm{~N}=3$ & $\mathrm{~N}=10$ & $\mathrm{~N}=1$ & $\mathrm{~N}=3$ & $\mathrm{~N}=10$ \\
\hline Optimal & 164.32 & 487.03 & 1615.52 & 150.14 & 450.09 & 1499.60 \\
C-Blind & 178.79 & 536.37 & 1787.90 & 164.10 & 492.30 & 1641.00 \\
UCTR1 & 164.32 & 487.36 & 1618.00 & 150.14 & 450.25 & 1500.64 \\
UCTR2 & 164.32 & 487.13 & 1619.32 & 150.14 & 450.13 & 1500.06 \\
\hline
\end{tabular}

Table 2: Results for the set of 20 edges with 8 unknown

show significant improvements over the followers polices UCTR1 and UCTR2. Since the time spent to compute the reasoning policies is much larger than the followers polices, we conclude that these versions are not recommended. This bolsters our theoretical results, which show that following is optimal on disjoint paths: while not optimal in general, it is a strong heuristic. We thus only report the total cost of the followers polices UCTR1 and UCTR2.

Table 2 presents the total costs incurred on our graph (with 8 unknown edges) by the two benchmark algorithms and by the followers policies UCTR1 and UCTR2 when the number of agents $n$ was varied. We experimented with several instances and present results on two representative instances. It is important to observe that both UCTR1 and UCTR2 are very close to the optimal policy, and are far better than the cautious-blind bound. This strengthen our claim that the optimal behavior for the disjoint-path graphs is a strong heuristic for general graphs.

When the number of agents $n$ is small, UCTR2 slightly outperforms UCTR1. When $n$ is larger (10 agents), results are inconclusive. As indicated by varying the number of rollouts (not shown), the reason is that with many agents the "considerate" assumption and the large extra $F^{k}$ term in the UCTR formula (especially as it is multiplied by the number of remaining agents) amplifies the sampling noise. This noise is hard to overcome unless the number of rollouts is increased drastically.

\section{Discussion}

UCTR2 outperforms UCTR1 if some policy $\Pi_{c}$ exists (c for considerate) such that using $\Pi_{c}$ on CTP-REP has lower expected cost than taking the "selfish" policy $\Pi_{s}$ where each agent tries to minimize it's own cost function. We expect UCTR2 to resolve with $\Pi_{c}$ and UCTR1 to resolve with $\Pi_{s}$

Denote $\Pi^{1}$ as the policy that the first agent takes and $\Pi^{F}$ as the "followers" policy, used by the rest of the agents. In general, $E\left[\Pi_{s}^{1}\right] \leq E\left[\Pi_{c}^{1}\right]$ since $\Pi_{s}^{1}$ tries to minimize the cost function without the burden of the follower agents.

Despite this fact, UCTR2 will outperforms UCTR1 when:

$$
E\left[\Pi_{c}^{1}\right]+(n-1) \times E\left[\Pi_{c}^{F}\right]<E\left[\Pi_{s}^{1}\right]+(n-1) \times E\left[\Pi_{s}^{F}\right] .
$$

The graph in figure 2(b) demonstrates such a case, and indeed UCTR2 outperforms UCTR1 when the number of agents is large enough. In this instance, all edges are known except the dashed edge which has blocking probability of 0.95 .

On the initial state $s, \Pi^{1}(s)$ chosen by UCTR1 results in taking action $a_{1}$ (traverse $a_{1}$ ) with cost 100 since taking the alternative action $a_{2}$ evaluates to $0.05 \times 50+0.95 \times$ $150=145$. The follower policy $\Pi^{F}(s)$ also follows $a_{1}$ and evaluates to 100 .

Now, let's consider UCTR2. If $\Pi^{1}(c)$ chooses to take $a_{1}$ then the follower policy, $\Pi^{F}(s)$ evaluates to 100 , as in UCTR1. However if $\Pi^{1}(c)$ takes $a_{2}$ then the value of $\Pi^{F}(c)$ will be $0.95 \times 100+0.05 \times 50=97.5$ which is slightly better for the followers. It is beneficial for UCT2 to choose $\Pi^{1}(c)$ to be $a_{2}$ when the extra cost incurred by $\Pi^{1}(c)$ is compensated by the advantage gained by the followers. Therefore, it is beneficial to take $a_{2}$ only when $(n-1) \times(100-97.5)>145-100$. For our case, it happens for $n \geq 20$.

We also experimented with instances that have large number of unknown edges (up to 20). In this case, evaluating the policy against all weathers is not practical therefor only a subset of weathers is used. In these experiments we get inconclusive results - on some cases UCTR-1 seems to outperform UCTR-2 probably. In such cases, although the above condition holds, experimenting with only a subset of weathers does not necessarily demonstrate the benefit of using UCTR2 because of the negative skewness of $E\left[\Pi_{c}^{2}\right]$.

\section{Conclusions}

Repeated task CTP is introduced, and an optimal followers policy for the special case of disjoint-path graphs is defined and proved. This followers policy forms the basis of heuristics for reasonable non-optimal policies for general graphs, as suggested by an empirical evaluation on using a suite of sampling algorithms based on UCT. While optimal for disjoint-path graphs, the followers policy is very strong and is near optimal for general graphs.

The more general interleaved-agent CTP was also introduced. However, this problem is more complicated due to the much larger state and action spaces. For disjoint-path graphs, empirical results indicate that the followers policy is optimal, and that there is nothing to gain by allowing interleaved actions. Proving (or disproving) this conjecture would be a good way to continue research on this CTP variant. Experimental results demonstrate cases where UCTR1 outperformed UCTR2.

\section{Acknowledgement}

This work was supported by ISF grant \#305/09

\section{References}

Bar-Noy, A., and Schieber, B. 1991. The Canadian Traveller Problem. In SODA, 261-270.

Bnaya, Z.; Felner, A.; and Shimony, S. E. 2009. Canadian traveler problem with remote sensing. In IJCAI, 437-442.

Bonet, B. 2009. Deterministic POMDPs Revisited. In (UAI). Montreal, Canada.

Eyerich, P.; Keller, T.; and Helmert, M. 2010. High-Quality Policies for the Canadian Traveler's Problem. In AAAI 2010.

Hansen, E. A. 2007. Indefinite-horizon POMDPs with actionbased termination. In AAAI, 1237-1242.

Kocsis, L., and Szepesvári, C. 2006. Bandit based monte-carlo planning. In ECML, 282-293.

Littman, M. 1996. Algorithms for Sequnetial Decision Making. Ph.D. Dissertation, Brown University.

Meshulam, R.; Felner, A.; and Kraus, S. 2005. Utility-based multiagent system for performing repeated navigation tasks. In $A A M A S$, 887-894.

Nikolova, E., and Karger, D. R. 2008. Route Planning under Uncertainty: The Canadian Traveller Problem. AAAI 969-974.

Papadimitriou, C. H., and Yannakakis, M. 1991. Shortest Paths Without a Map. Theor. Comput. Sci. 84(1):127-150. 